\title{
Papillary Cystadenoma of the Fallopian Tube Not Associated with von Hippel-Lindau Disease: A Case Report
}

\author{
Jae Yeon Seok • Myunghee Kang · Jungsuk An · Hyunchul Kim · Kwang-Beom Lee ${ }^{1} \cdot$ Hyun Yee Cho \\ Departments of Pathology and ${ }^{1}$ Obstetrics and Gynecology, Gachon University Gil Medical Center, Incheon, Korea
}

Papillary cystadenoma (PC) is an epithelial tumor believed to be of mesonephric origin ${ }^{1}$ that is found rarely in the female genital tract. Since the original report by Gersell and King in 1988, ${ }^{2}$ only 14 cases have been reported in the English literature..$^{1,-3}$ All of the reported cases occurred in the mesosalpinx or the broad ligament. Most of the tumors (12 out of 14) were associated with von Hippel-Lindau disease (VHL), similar to epididymal tumors. ${ }^{6}$ We report a case of $\mathrm{PC}$ within the wall of the fallopian tube (rather than in the mesosalpinx or the broad ligament) in a patient with no clinical history of VHL. A comprehensive review of the literature with regard to differential diagnosis and immunohistochemical profile is provided.

\section{CASE REPORT}

A 44-year-old multipara woman presented with a complaint of a menorrhagia for six months. She and her family had no history of VHL. Ultrasonogram showed multiple solid masses on the uterus, suggesting leiomyomas. The laboratory findings were unremarkable, except for a slightly decreased hemoglobin level $(11.7 \mathrm{~g} / \mathrm{dL})$. Laparoscopy was performed under the diagnosis of uterine leiomyomas. Both fallopian tubes were enlarged and showed adhesion to the uterine serosa, colonic serosa, and pelvic wall. Total hysterectomy with bilateral salpingectomy and adhesiolysis was performed.

The distal half of the left fallopian tube was slightly enlarged

\section{Corresponding Author}

Hyun Yee Cho, M.D.

Department of Pathology, Gachon University Gil Medical Center, 21 Namdong-daero

774beon-gil, Namdong-gu, Incheon 405-760, Korea

Tel: +82-32-460-3073, Fax: +82-32-460-2394, E-mail: hicho@gilhospital.com

Received: August 6, 2013 Revised: October 18, 2013

Accepted: October 24, 2013 and the outer surface was smooth. On section, a solid grayishyellow mass that measured $3.9 \times 1.4 \times 1.3 \mathrm{~cm}$ was observed along the long axis of the fallopian tube (Fig. 1). On microscopic examination, a papillary cystic tumor was noted within the wall of the fallopian tube pushing against the surface epithelium. The papillae were short and blunted and lined by a single layer of bland nonciliated cuboidal cells with clear to eosinophilic cytoplasm. The nuclei were round with fine chromatin and occasional inconspicuous nucleoli (Fig. 2). Psammoma bodies and mitotic figures were not found. There was a focus of endometriosis at the adhesion site. The resected uterus contained multiple leiomyomas and the myometrium showed adenomyosis. The right fallopian tube showed chronic inflammation.

Immunohistochemical examination was performed according to a previously reported method. ${ }^{7}$ The tumor cells showed strong positivity for Cam5.2, cytokeratin (CK) 7, high-molecular weight $\mathrm{CK}$, vimentin, and $\mathrm{CD} 10$ (Fig. 3) and weak positivity for CK19 and epithelial membrane antigen (EMA). The tumor cells were negative for $\alpha$-methylacyl coenzyme A racemase, $\alpha$-inhibin, calretinin, c-kit, actin, and human melanoma black 45.

After the diagnosis of PC, abdominal computed tomography was performed, and revealed cysts in the liver and the left kidney. The patient was followed for seven months with no evidence of other clinical features of VHL.

\section{DISCUSSION}

PC of the female genital tract is a rare neoplasm. Since the original report in 1988, only 15 cases, including the current case, have been reported in the English literature (Table 1). ${ }^{2}$ The patients ranged in age from 20 to 56 years $(37.9 \pm 11.0$ years). Nine of 14 cases $(60 \%)$ occurred at the mesosalpinx and six of 


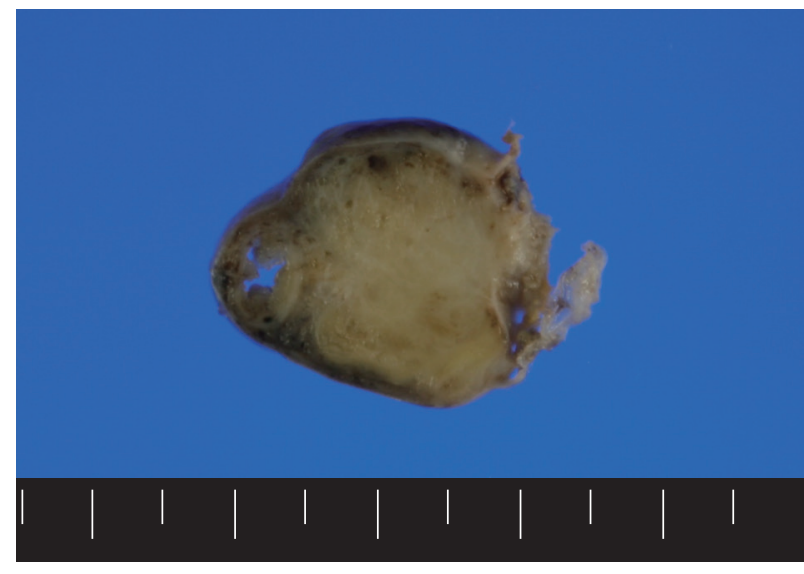

Fig. 1. An ill-defined solid yellow-tan mass is noted in the fallopian tube wall. Grossly, the mass abuts the lumen of the fallopian tube.
$14(40 \%)$ at the broad ligament. No case of PC arising within the wall of the fallopian tube has previously been reported. In our case, the tumor was located within the wall of the fallopian tube rather than in the mesosalpinx or broad ligament, but was not intraluminal. Ten of 11 previous cases were unilateral (six in the left and four in the right). The size of the tumors ranged from $3 \mathrm{~cm}$ to $6.5 \mathrm{~cm}(4.6 \pm 1.2 \mathrm{~cm})$. Twelve of 14 cases $(86 \%)$ were associated with VHL either clinically or genetically. Seven of those had a previous history of VHL and PC was detected under close follow-up and radiologic study. In five patients, the tumor was discovered as an incidental finding with no history of VHL before surgery and clinical investigation revealed the presence of VHL after diagnosis. In three of 15 cases, including the current case, the PC was an incidental finding and there was no identifiable evidence of VHL, despite clinical investigation
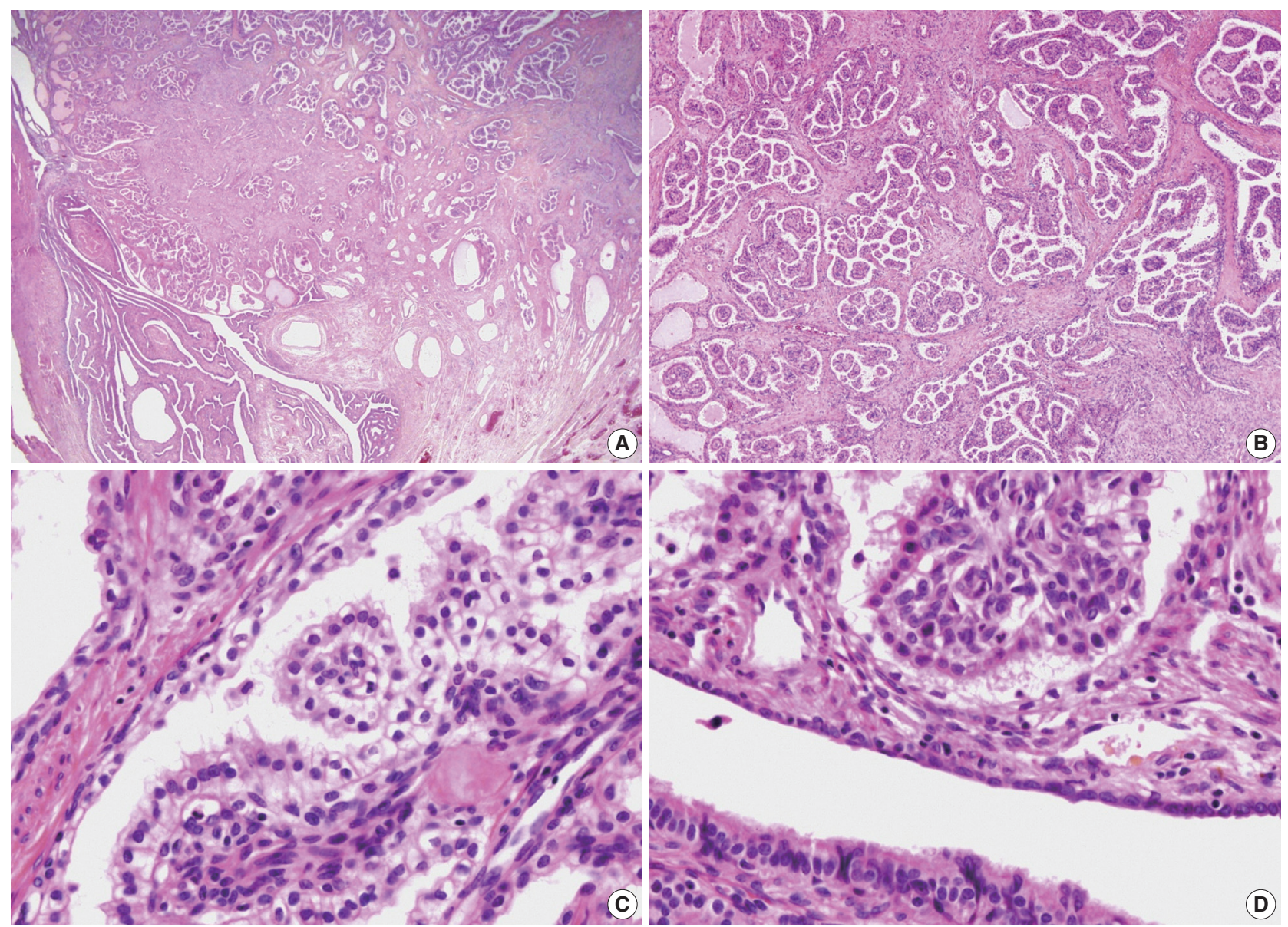

B.

Fig. 2. Low-power view of papillary cystadenoma shows an expansile growth pushing the lumen of the fallopian tube and blunting the plicae of the fallopian tube (lower left) (A). The tumor has many small cystic spaces containing papillary projections with glomeruloid features in some areas (B). The papillary fronds have monolayered cuboidal/columnar epithelium showing clear cytoplasm and a distinct cell border. The fibrovascular core contains a compact capillary network (C). Tumor cells with eosinophilic cytoplasm do not have cilia compared to ciliated tubal epithelial cells (lower left) (D). 

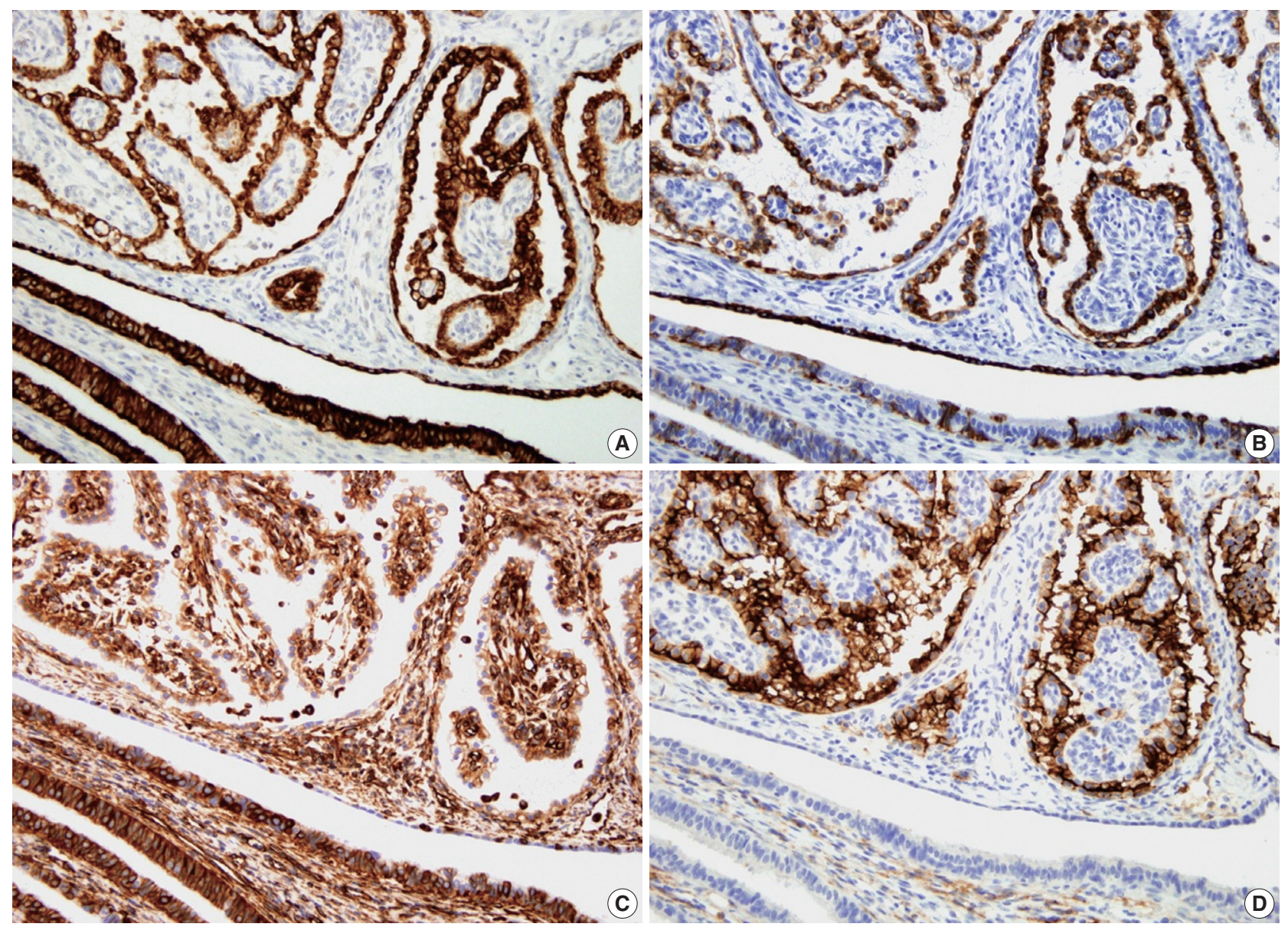

Fig. 3. Immunohistochemically, the tumor cells show positivity for Cam5.2 (A), cytokeratin 7 (CK7) (B), vimentin (C), and CD10 (D). In contrast, the epithelial cells of the fallopian tube (lower left) show strong positivity for Cam5.2 and vimentin, variable positivity for CK7, and negativity for CD10.

after the diagnosis. Thirteen of 14 cases showed benign courses without recurrence or metastasis after surgery. There was one case of recurrence after incomplete resection. ${ }^{3}$ In the other case, the tumor showed two foci of omental implant, which led to overdiagnosis of malignant tumor on frozen section. ${ }^{4}$ Adhesion with the serosa of the intestine was observed in two cases. ${ }^{5}$

A summary of the immunohistochemical findings of previously reported cases and the current case is provided in Table 2. PC typically expresses $\mathrm{CK} 7$, pan-CK, and low-molecular weight CK (Cam5.2). CD10, EMA, and vimentin are often positive but may be negative. These findings favor mesonephric rather than Müllerian origin.

Differential diagnosis of PC should include metastatic clear cell renal cell carcinoma (RCC), cystadenofibroma of the Müllerian type, atypical proliferative serous tumor, and papillary syncytial metaplasia. PCs, which are predominantly of clear cell type, can mimic metastatic RCC especially in a patient with a history of VHL. In contrast with RCC, PC shows diffuse ex- pression of $\mathrm{CK} 7$ and is usually negative for RCC markers (Table 2). ${ }^{3}$ In cystadenofibroma of Müllerian type, the papillae are much larger and less complex and contain cilia. In atypical proliferative serous tumors, the papillae show hierarchical branching with epithelial stratification, ciliated cells, and psammoma bodies. The tumor cells in our case showed little cytologic atypia, no cilia, and no mitotic figure. Papillary syncytial metaplasia of tubal epithelium is typically associated with endometriosis and should be included in the differential diagnosis. In papillary syncytial metaplasia the metaplastic epithelium shows dense eosinophilic syncytial cytoplasm in contrast to clear cytoplasm with distinct cell borders as in this case.

Due to its rarity, the diagnosis of PC arising in the female genital tract is often challenging in daily practice. The diagnosis on frozen section can be more difficult since the cytologic details are obscured by the artifact. The awareness of this entity can prevent misdiagnosis.

Due to its strong association with VHL, the accurate diagno- 
Table 1. Clinical features of papillary cystadenoma in the female genital tract

\begin{tabular}{|c|c|c|c|c|c|c|c|c|c|}
\hline Case No. & Year & Age (yr) & Site & Side & Size $(\mathrm{cm})$ & Symptom & History of VHL & VHL & Other \\
\hline $1^{2}$ & 1988 & 46 & MS & $\mathrm{Lt}$ & 3 & None & $P(20 \mathrm{yr})$ & $\begin{array}{l}\text { ReA, RCC, } \\
\text { KLL/P cyst }\end{array}$ & Fimbrial adhesion \\
\hline 2 & 1989 & 46 & $\mathrm{BL}$ & $\mathrm{Lt}$ & 3 & Mass & $P(25 y r)$ & - & - \\
\hline 3 & 1990 & 35 & $\mathrm{BL}$ & Rt & 5 & Pain & A & $\begin{array}{l}\mathrm{CbH}, \mathrm{RCC} \\
\text { K/P cyst }\end{array}$ & - \\
\hline $4^{1}$ & 1994 & 20 & $\mathrm{BL}$ & Rt & 4 & Nausea & A & $\begin{array}{l}\mathrm{CbH}, \mathrm{RCC} \\
\text { ELST, K/P cyst }\end{array}$ & - \\
\hline $5^{3}$ & 2005 & 32 & MS & NA & NA & NA & $P$ & RCC & - \\
\hline $6^{3}$ & 2005 & NA & MS & NA & NA & NA & A & - & Misdiagnosis (serous borderline tumor) \\
\hline 7 & 2010 & 23 & MS & Rt & 3.5 & Pain & $P(5 y r)$ & $\mathrm{SpH}$ & - \\
\hline $8^{4}$ & 2012 & 52 & MS & Lt & 6 & Pain & N & - & $\begin{array}{c}\text { Omental implant } \\
\text { Misdiagnosis (malignant serous tumor) }\end{array}$ \\
\hline $9^{4}$ & 2012 & 35 & $\mathrm{BL}$ & NA & 3.5 & None & $\mathrm{P}$ & - & - \\
\hline $10^{4}$ & 2012 & 34 & MS & Rt & 5.5 & None & $A$ & - & - \\
\hline $11^{4}$ & 2012 & 24 & MS & $\mathrm{Lt}$ & 5 & None & $A$ & - & - \\
\hline $12^{4}$ & 2012 & 36 & MS & Bilat & Rt 5.2, Lt 3 & None & $\mathrm{P}$ & - & - \\
\hline $13^{5}$ & 2012 & 47 & $\mathrm{BL}$ & $\mathrm{Lt}$ & 6.5 & None & $\mathrm{P}$ & $\mathrm{CbH}$ & Adhesion to rectum \\
\hline $14^{5}$ & 2012 & 56 & PD & & 6 & Pain & $\mathrm{N}$ & - & - \\
\hline 15 & Present case & 44 & MS & $\mathrm{Lt}$ & 3.9 & None & $\mathrm{N}$ & K/L cyst & Fallopian tube involvement \\
\hline
\end{tabular}

VHL, von Hippel-Lindau disease; MS, mesosalpinx; Lt, left; P, previous history of VHL; ReA, retinal angioma; RCC, renal cell carcinoma; KLLP, kidney/liver/ pancreas; BL, broad ligament; Rt, right; A, VHL detected after the diagnosis of papillary cystadenoma; $\mathrm{CbH}$, cerebellar hemangioblastoma; ELST, endolymphatic sac tumor; NA, not available; $\mathrm{SpH}$, spinal cord hemangioblastoma; N, no previous history and no further detection of VHL after the diagnosis of papillary cystadenoma; Bilat, bilateral; PD, pouch of Douglas.

Table 2. Immunohistochemical findings of papillary cystadenoma in the female genital tract

\begin{tabular}{lccc}
\hline Marker & Present case & 14 Reported cases & Positive rate (\%) \\
\hline CK7 & + & $10 / 10$ & 100 \\
Cam5.2 & + & $6 / 6$ & 100 \\
HMW-CK & + & $1 / 1$ & 100 \\
CK & ND & $5 / 5$ & 100 \\
CK19 & + & $1 / 1$ & 100 \\
CK20 & ND & $0 / 4$ & 0 \\
Ber-EP4 & ND & $3 / 3$ & 100 \\
EMA & + & $9 / 11$ & 81.8 \\
Vimentin & + & $8 / 9$ & 88.9 \\
CD10 & + & $9 / 10$ & 90 \\
a-Inhibin & - & $0 / 3$ & 0 \\
Calretinin & - & $3 / 8$ & 37.5 \\
AMACR & - & $0 / 11$ & 0 \\
HMB45 & - & $0 / 1$ & 0 \\
C-kit & - & $0 / 1$ & 0 \\
PAX2 & ND & $5 / 5$ & 100 \\
RCC marker & ND & $1 / 9$ & 11.1 \\
VHL40 & ND & $3 / 5$ & 60 \\
CA125 & ND & $5 / 7$ & 71.4 \\
WT-1 & ND & $4 / 7$ & 57.1 \\
HBME-1 & ND & $2 / 5$ & 40 \\
CEA & ND & $0 / 3$ & 0 \\
ER & ND & $0 / 2$ & 0 \\
PR & ND & $0 / 2$ & 0 \\
AR & ND & $2 / 7$ & 28.6 \\
\hline CK, cytok & & & \\
\hline
\end{tabular}

$\mathrm{CK}$, cytokeratin; HMW-CK, high-molecular weight cytokeratin; ND, not done; EMA, epithelial membrane antigen; AMACR, a-methylacyl coenzyme A racemase; HMB45, human melanoma black 45; RCC, renal cell carcinoma; VHL40, von Hippel-Lindau 40; CA125, cancer antigen 125; WT-1, Wilms tumour-1; CEA, carcinoembryonic antigen; ER, estrogen receptor; PR, progesterone receptor; AR, androgen receptor. sis of PC is important. If the diagnosis of PC is made in a patient not known to have VHL, clinical investigations should be performed in order to confirm or rule out the possibility of VHL. Early diagnosis of VHL can enable the identification of potentially life-threatening lesions, including RCC, at curable stages.

\section{Conflicts of Interest}

No potential conflict of interest relevant to this article was reported.

\section{REFERENCES}

1. Gaffey MJ, Mills SE, Boyd JC. Aggressive papillary tumor of middle ear/temporal bone and adnexal papillary cystadenoma: manifestations of von Hippel-Lindau disease. Am J Surg Pathol 1994; 18: 1254-60.

2. Gersell DJ, King TC. Papillary cystadenoma of the mesosalpinx in von Hippel-Lindau disease. Am J Surg Pathol 1988; 12: 145-9.

3. Aydin H, Young RH, Ronnett BM, Epstein JI. Clear cell papillary cystadenoma of the epididymis and mesosalpinx: immunohistochemical differentiation from metastatic clear cell renal cell carcinoma. Am J Surg Pathol 2005; 29: 520-3.

4. Nogales FF, Goyenaga P, Preda O, et al. An analysis of five clear cell papillary cystadenomas of mesosalpinx and broad ligament: four associated with von Hippel-Lindau disease and one aggressive sporadic type. Histopathology 2012; 60: 748-57.

5. Brady A, Nayar A, Cross P, et al. A detailed immunohistochemical 
analysis of 2 cases of papillary cystadenoma of the broad ligament: an extremely rare neoplasm characteristic of patients with von hippel-lindau disease. Int J Gynecol Pathol 2012; 31: 133-40.

6. Chun YK, Lee WA, Khang SK. Papillary cystadenoma of the epididymis: component of von Hippel-Lindau syndrome: a case re- port. Korean J Pathol 1994; 28: 663-5.

7. Jang TJ, Park JB, Lee JI. The Expression of CD10 and CD15 is progressively increased during colorectal cancer development. Korean J Pathol 2013; 47: 340-7. 